Article

\title{
A Sensitive LC-MS/MS Method for the Quantification of 3-Hydroxybenzo[a]pyrene in Urine-Exposure Assessment in Smokers and Users of Potentially Reduced-Risk Products
}

\author{
Nadine Rögner, Heinz-Werner Hagedorn, Gerhard Scherer, Max Scherer and Nikola Pluym *(D) \\ Analytisch-Biologisches Forschungslabor GmbH, Semmelweisstr. 5, 82152 Planegg, Germany; \\ nadine.roegner@abf-lab.com (N.R.); info@abf-lab.com (H.-W.H.); gerhard.scherer@abf-lab.com (G.S.); \\ max.scherer@abf-lab.com (M.S.) \\ * Correspondence: pluym@abf-lab.com; Tel.: +49-89-4114796-12
}

\section{check for}

updates

Citation: Rögner, N.;

Hagedorn, H.-W.; Scherer, G.;

Scherer, M.; Pluym, N. A Sensitive

LC-MS/MS Method for the

Quantification of 3-Hydroxybenzo[a] pyrene in Urine-Exposure

Assessment in Smokers and Users of Potentially Reduced-Risk Products. Separations 2021, 8, 171. https://doi. org/10.3390/separations 8100171

Academic Editor: Fadi Aldeek

Received: 8 September 2021

Accepted: 29 September 2021

Published: 5 October 2021

Publisher's Note: MDPI stays neutral with regard to jurisdictional claims in published maps and institutional affiliations.

Copyright: (c) 2021 by the authors. Licensee MDPI, Basel, Switzerland. This article is an open access article distributed under the terms and conditions of the Creative Commons Attribution (CC BY) license (https:// creativecommons.org/licenses/by/ $4.0 /)$.
Abstract: Benzo[a]pyrene (BaP), a human carcinogen, is formed during the incomplete combustion of organic matter such as tobacco. A suitable biomarker of exposure is the monohydroxylated metabolite 3-hydroxybenzo[a]pyrene (3-OH-BaP). We developed a sensitive LC-MS/MS (liquid chromatography coupled with tandem mass spectrometry) method for the quantification of urinary 3-OH-BaP. The method was validated according to the US Food and Drug Administration (FDA) guideline for bioanalytical method validation and showed excellent results in terms of accuracy, precision, and sensitivity (lower limit of quantification (LLOQ): $50 \mathrm{pg} / \mathrm{L}$ ). The method was applied to urine samples derived from a controlled clinical study to compare exposure from cigarette smoking to the use of potentially reduced-risk products. Urinary 3-OH-BaP concentrations were significantly higher in smokers of conventional cigarettes $(149 \mathrm{pg} / 24 \mathrm{~h}$ ) compared to users of potentially reducedrisk products as well as non-users ( $99 \%<$ LLOQ in these groups). In conclusion, 3-OH-BaP is a suitable biomarker to assess the exposure to $\mathrm{BaP}$ in non-occupationally exposed populations and to distinguish not only cigarette smokers from non-smokers but also from users of potentially reduced-risk products.

Keywords: 3-hydroxybenzo[a]pyrene; LC-MS/MS; urine; human biomonitoring; derivatization; potentially reduced-risk products

\section{Introduction}

Polycyclic aromatic hydrocarbons (PAHs) are formed during the incomplete combustion of organic matter. High exposures are observed at special workplaces such as cookeries, steel factories, and road buildings. Exposure of the general population to PAHs is mainly caused by environmental factors such as polluted air and water, by the consumption of smoked and grilled food, and by smoking of conventional (combustible) cigarettes (CC), respectively [1-6].

Over the past decade, several new nicotine and tobacco products have been introduced as alternatives to smoking with a potentially reduced health risk compared to CC. As many PAHs are carcinogenic due to their metabolic activation of DNA reactive compounds, the measurement of specific biomarkers is of great importance to assess the exposure to PAHs from potentially reduced-risk products.

For the determination of PAH exposure, usually, respective monohydroxylated urinary metabolites are analyzed by means of LC-MS/MS (liquid chromatography coupled with tandem mass spectrometry) or GC-MS (gas chromatography-mass spectrometry). For instance, 1-hydroxypyrene, monohydroxy-fluorenes, and monohydroxy-phenanthrenes are frequently determined in urine samples in order to investigate exposure to PAHs [7-10]. Benzo[a]pyrene $(\mathrm{BaP}$, Figure 1$)$ is classified as a Group 1 carcinogen (carcinogenic to 
humans) by the International Agency for Research on Cancer (IARC) and is by now the best-studied PAH [3,11].<smiles>c1ccc2c(c1)cc1ccc3cccc4ccc2c1c34</smiles><smiles>Oc1ccc2ccc3c4ccccc4cc4ccc1c2c43</smiles>

Figure 1. Chemical structure of benzo[a]pyrene (BaP, left) and its metabolite 3-hydroxybenzo[ $[a]$ pyrene (3-OH-BaP, right).

A key metabolite of $\mathrm{BaP}$ is (+)-anti-BaP-7,8-diol-9,10-epoxide (BPDE), which is considered as an ultimate carcinogen, reacting with cellular DNA, proteins, and glutathione. Furthermore, BPDE can react by enzymatic hydrolysis to form BaP- $(7,8,9,10)$-tetrol, which is excreted in the urine after conjugation with, e.g., glucuronic acid [11-15]. This biomarker, therefore, found use in studying exposure to $\mathrm{BaP}[16,17]$. Very low concentration levels require laborious sample preparation to achieve sufficient sensitivity of the analytical methods, making routine analysis very challenging for this biomarker.

An alternative biomarker of $\mathrm{BaP}$ exposure is the monohydroxylated metabolite 3hydroxybenzo[a]pyrene (3-OH-BaP, Figure 1), which is excreted in urine after conjugation. Nearly $100 \%$ of the urinary $3-\mathrm{OH}-\mathrm{BaP}$ detected in humans is excreted as glucuronide or sulfate [18].

Several methods have been developed and established for the determination of 3-OH$\mathrm{BaP}$ in urine for occupationally exposed subjects [19-23]. However, those methods are limited by the lack of sensitivity to determine the burden of $\mathrm{BaP}$ exposure in the general population. In order to cover not only occupational but also environmental exposure, including cigarette smoking, sensitivity in the pg/L-range is required. This can be achieved by optimizing the sample preparation, including derivatization steps [24-27], purification and concentration procedures [28-30], or by application of different ionization techniques, such as atmospheric pressure laser ionization (APLI) [31]. Thus, many of these methods are hampered by complex analytical procedures and specific/expensive equipment that can only be used to a limited extent in larger cohorts of human biomonitoring campaigns and clinical studies, respectively.

The aims of the current study were to adjust and validate a sensitive and robust method for the quantitation of 3-OH-BaP in urine with a sufficiently high sample throughput. Further, the validated method was applied to urine samples collected in a controlled clinical trial [32] with 10 users per group of 5 different nicotine-containing products, including smokers of conventional cigarettes (CC), users of electronic cigarettes (EC), users of heated tobacco products (HTP), users of nicotine replacement therapy (NRT), users of oral tobacco (OT), and non-users (NU), in order to distinguish differences in the exposure from these products.

\section{Materials and Methods}

\subsection{Chemicals}

3-Hydroxybenzo[a]pyrene-O- $\beta$-glucuronide (3-OH-BaP-Gluc, molecular weight (MW): $444 \mathrm{~g} / \mathrm{mol}$ ), 3-OH-BaP (MW: $268 \mathrm{~g} / \mathrm{mol}$ ), and ${ }^{13} \mathrm{C}_{6}-3-\mathrm{OH}-\mathrm{BaP}-\mathrm{Gluc}$ were purchased from AptoChem (Montreal, QC, Canada). ${ }^{2} \mathrm{H}_{11}-3-\mathrm{OH}-\mathrm{BaP}$ was purchased from TRC (Toronto, ON, Canada). N,N-dimetylethylamine (DMEA) was obtained from Alfa Aesar (Karlsruhe, Germany), 2-fluoro-methylpyridinium-p-toluenesulfonate (FMPT) from TCI (EsMVchborn, Germany), formic acid 99\%, ULC/MS grade from Biosolve (Valkenswaard, The Netherlands), and acetic acid, ascorbic acid, dimethyl sulfoxide, hydrochloric acid 37\%, and sodium hydroxide from Merck (Darmstadt, Germany). Dichloromethane and methanol for residue analysis and LC-MS grade acetonitrile and methanol were purchased from LGC Standards (Wesel, Germany). Water was purified by means of a Sartorius arium 
water system (Göttingen, Germany). The enzyme $\beta$-glucuronidase/arylsulfatase from Helix pomatia (4.5 and $14 \mathrm{U} / \mathrm{mL}$ ) was supplied by Roche (Mannheim, Germany).

\subsection{Sample Work-Up for Quantification}

For sample preparation, the work-up published previously [25] was applied with major modifications. Frozen urine samples were thawed slowly at room temperature. To homogenized urine $(6 \mathrm{~mL})$, acetate buffer $(400 \mu \mathrm{L} ; 1 \mathrm{M}, \mathrm{pH}=5.1)$ was added, and the $\mathrm{pH}$-value of the sample was adjusted with hydrochloric acid $(1 \mathrm{~N})$ to $\mathrm{pH} 5.0-5.5$ if necessary. Aliquots $(100 \mu \mathrm{L})$ of an aqueous solution of the internal standard were added, containing an absolute amount of $10 \mathrm{pg}{ }^{13} \mathrm{C}_{6}-3-\mathrm{OH}-\mathrm{BaP}-\mathrm{Gluc}$, followed by the addition of $100 \mu \mathrm{L}$ of ascorbic acid solution in water $(150 \mathrm{mg} / \mathrm{mL})$. For enzymatic hydrolysis, $\beta$-glucuronidase/arylsulfatase from Helix pomatia $(20 \mu \mathrm{L})$ was added, and the mixture was incubated overnight $(\sim 16-18 \mathrm{~h})$ at $37^{\circ} \mathrm{C}$. After incubation, samples were centrifuged (3000 rpm, $10 \mathrm{~min}$ ), and the supernatant was decanted into a new vessel and subjected to solid-phase extraction (SPE).

The SPE cartridges (Bond Elut-LMS, 200 mg, 3 mL; Agilent, Waldbronn, Germany) were conditioned with $3 \mathrm{~mL}$ of dichloromethane, $2 \times 3 \mathrm{~mL}$ of methanol, and $3 \mathrm{~mL}$ of water. Subsequently, the hydrolyzed urine mixture was added to the column. The tubes were washed with $3 \mathrm{~mL}$ of water, $3 \mathrm{~mL}$ of water/methanol (50/50, v/v), $1 \mathrm{~mL}$ of methanol, and $2 \mathrm{~mL}$ of methanol/acetonitrile $(50 / 50, \mathrm{v} / \mathrm{v})$. The target compound and internal standard were eluted with $2 \times 2 \mathrm{~mL}$ of dichloromethane in a $4 \mathrm{~mL}$ glass vial. To the eluate, dimethyl sulfoxide $(20 \mu \mathrm{L})$ was added, and dichloromethane was evaporated in a SpeedVac centrifuge (Thermo Fisher, Dreieich, Germany) without heating to a final volume of $20 \mu \mathrm{L}$ (containing only the dimethyl sulfoxide portion).

The residue was taken up in $250 \mu \mathrm{L}$ of FMPT solution $(0.5 \mathrm{mg} / \mathrm{mL}$ in acetonitrile) and $50 \mu \mathrm{L}$ of DMEA ( $0.2 \%$ in acetonitrile). The mixture was homogenized with a vortex mixer, and derivatization of the hydroxyl group was achieved by incubation of the mixture for $20 \mathrm{~min}$ at $45^{\circ} \mathrm{C}$. Samples were transferred to a microvial $(300 \mu \mathrm{L})$, and the solvent was evaporated in a SpeedVac centrifuge (Thermo Fisher, Dreieich, Germany) without heating to a final volume of $20 \mu \mathrm{L}$ (containing only the dimethyl sulfoxide portion). The residue was reconstituted in $250 \mu \mathrm{L}$ of methanol/water/formic acid (50/49/1, v/v/v) and homogenized with a vortex mixer. The extracts were analyzed by LC-MS/MS.

\section{3. $L C-M S / M S$}

An Agilent 1200 HPLC (Agilent, Waldbronn, Germany) was equipped with an Acquity UPLC BEH C18 column, $50 \times 2.1 \mathrm{~mm}$ i.d., $1.7 \mu \mathrm{m}$ (Waters, Eschborn, Germany) and coupled with a triple quadrupole mass spectrometer (API 5000; Sciex, Darmstadt, Germany). The injection volume was set to $15 \mu \mathrm{L}$. Chromatography was performed at a column temperature of $50{ }^{\circ} \mathrm{C}$ and at a flow rate of $0.6 \mathrm{~mL} / \mathrm{min}$. Solvent A (water with $0.5 \%$ formic acid) and solvent $B$ (acetonitrile with $0.5 \%$ formic acid) were used for elution. The gradient was 0-1 $\mathrm{min}, 20 \% \mathrm{~B}$; $1-7 \mathrm{~min}, 20-40 \% \mathrm{~B} ; 7-8.5 \mathrm{~min}, 40 \% \mathrm{~B} ; 8.5-10 \mathrm{~min}, 40-90 \% \mathrm{~B}$; $10-13 \mathrm{~min}, 90 \% \mathrm{~B} ; 13-13.1 \mathrm{~min}, 90-20 \% \mathrm{~B} ; 13.1-15 \mathrm{~min}, 20 \% \mathrm{~B}$. The ion source was operated in electrospray ionization (ESI)-positive mode. Nitrogen was used as the carrier gas. Source parameters were as follows: ion spray voltage, $5500 \mathrm{~V}$; source temperature, $680^{\circ} \mathrm{C}$; entrance potential, $10 \mathrm{~V}$; curtain gas, $30 \mathrm{psi}$; ion source gas 1, $50 \mathrm{psi}$; and ion source gas 2, $70 \mathrm{psi}$. MS measurements were performed by multiple reaction monitoring (MRM) mode. Detailed information for the MRM transitions and MS/MS parameters are summarized in Table 1. For controlling all modules and for data analysis, Analyst 1.5.2 software (Sciex) was used. 
Table 1. Retention times, mass transitions, dwell time, declustering potentials (DP), collision energies (CE), and cell exit potentials (CXP) for 3-OH-BaP and ${ }^{13} \mathrm{C}_{6}-3-\mathrm{OH}-\mathrm{BaP}$.

\begin{tabular}{cccccccc}
\hline Analyte or IS & $\begin{array}{c}\text { Retention Time } \\
(\mathbf{m i n})\end{array}$ & $\begin{array}{c}\text { Mass Transitions } \\
(\mathbf{m} / \mathbf{z})\end{array}$ & Role & Dwell Time (msec) & DP (V) & CE (V) & CXP (V) \\
\hline 3-OH-BaP & 6.9 & $360 \rightarrow 251$ & Quantifier & 150 & 161 & 45 \\
3-OH-BaP & 6.9 & $360 \rightarrow 267$ & Qualifier & 150 & 18 & 45 \\
${ }^{13} \mathrm{C}_{6}-3-\mathrm{OH}-\mathrm{BaP}$ & 6.9 & $366 \rightarrow 257$ & IS & 150 & 161 & 45 \\
\hline
\end{tabular}

\subsection{Calibration}

To determine the concentration of 3-OH-BaP in urine, a calibration line was generated in non-smoker urine (analyte-free) by spiking increasing amounts of 3-OH-BaP-Gluc to receive concentrations between 50 and $3321 \mathrm{pg} / \mathrm{L}$, based on free 3-OH-BaP, while the internal standard amount remained constant $\left(10 \mathrm{pg}{ }^{13} \mathrm{C}_{6}-3-\mathrm{OH}-\mathrm{BaP}-\mathrm{Gluc}\right)$. Calibrators were worked up as described above and analyzed by LC-MS/MS. The calibration line equation was obtained by linear regression (1/y weighting) of the area ratio (area counts of the analyte/area counts of the internal standard) and the spiked analyte concentration. The 3-OH-BaP concentration in human urine samples was then calculated from the area counts ratios between 3-OH-BaP and ${ }^{13} \mathrm{C}_{6}-3-\mathrm{OH}-\mathrm{BaP}$ by employing the calibration line, equation with $y$ being the area count ratio and $x$ being nominal the $3-\mathrm{OH}-\mathrm{BaP}$ concentration.

\subsection{Method Validation}

The method was validated according to the US Food and Drug Administration (FDA) guideline [33]. The method was initially developed and validated using the free forms of both the reference and the internal standard for quantification. Hence, analyte-free non-smoker urine was spiked with free 3-OH-BaP in different concentration levels (low, medium, and high) to cover the entire calibration range. As internal standard, ${ }^{2} \mathrm{H}_{11}-3-\mathrm{OH}-$ $\mathrm{BaP}$ was used during the initial method validation. All working solutions of the analyte and the standard were freshly prepared before use. As a consequence of the stability investigations, the final method comprises the glucuronides 3-OH-BaP-Gluc and ${ }^{13} \mathrm{C}_{6}-3-$ $\mathrm{OH}-\mathrm{BaP}$ instead of the free forms, as discussed in the Section 3. Additionally, ascorbic acid was added to protect the free 3-OH-BaP formed in the urine samples during enzymatic hydrolysis.

To monitor the accuracy and the precision during study sample analysis, internal quality control samples (QCs) were prepared by spiking analyte-free non-smoker urine with known concentrations of 3-OH-BaP-Gluc. The QCs, covering the expected concentration range (QC low, QC medium, QC high), were randomly interspersed with the study samples (min. 5\% of total sample size or at least two per level) during sample work-up and analysis. The acceptance criteria for the QCs were defined by accuracy of $85-115 \%$. The target values were previously determined by analyzing six QCs per level.

Selectivity was verified for the MRM transitions of the analyte (quantifier and qualifier) and the corresponding internal standard. Samples of six different analyte-free non-smoker urines were compared with a blank sample containing only the reference compounds, prepared and analyzed under the same conditions. Each transition was screened for potential interferences that had the same retention times as the analyte or the internal standard signal. The same six samples were spiked with $400 \mathrm{pg} / \mathrm{L} 3-\mathrm{OH}-\mathrm{BaP}$ and analyzed for accuracy $(85-115 \%)$ and precision $(\mathrm{CV} \leq 15 \%)$.

The LLOQ (lower limit of quantification) was determined by analyzing five replicates of spiked non-smoker urine at the lowest concentration $(50 \mathrm{pg} / \mathrm{L})$, achieving a precision of at least $20 \%$ and an accuracy rate of $80-120 \%$. The LOD (limit of detection) was then obtained by dividing the LLOQ by 3 .

Accuracy and precision were determined by spiking non-smoker urine at different concentration levels (LLOQ, low, medium, and high). Inter-day accuracy and precision were determined by analyzing five spiked urine samples per level on three different 
days. Intra-day accuracy and precision were obtained from the analysis of one day. The acceptance criteria for intra-day and inter-day precision were specified by the calculation of coefficients of variation (CVs), which should be below $15 \%$ and $20 \%$, respectively, for concentrations below three times LLOQ. Accuracy rates should be in the range of $85-115 \%$ of nominal concentrations and $80-120 \%$ for concentrations below three times the LLOQ.

Recovery rates indicate analyte losses during sample work-up. The recovery rates were determined at three different concentration levels by comparing the analyte area of non-smoker urine samples spiked before sample work-up $(\mathrm{N}=6)$ and after SPE extraction $(\mathrm{N}=3)$ with free 3-OH-BaP. Samples spiked after SPE extraction correspond to $100 \%$ and served as reference.

The matrix effect (ME) was evaluated by comparing the signals of analyte and internal standard at two different concentration levels (low and high) of post-spiked (after SPE extraction) processed urine samples $(\mathrm{N}=3)$ with a sample of the reference standards. The relative difference to the reference signals $(100 \%)$ was defined as ME. Relative differences of $>0 \%$ indicate a positive ME (signal enhancement), and relative differences $<0 \%$ indicate a negative ME (signal suppression).

Carryover effects were analyzed by repeated injections $(\mathrm{N}=3 \times 5)$ of extracts spiked with high levels of the analyte $(2000 \mathrm{pg} / \mathrm{L})$ followed by the injection of a blank sample $(\mathrm{MeOH})$. No carryover effects were detected when the signal of the blank sample was at or below the LOD signal.

The stability of the analyte (free 3-OH-BaP or 3-OH-BaP-Gluc) was determined at room temperature for $24 \mathrm{~h}$ (short-term stability), at $10^{\circ} \mathrm{C}$ for $72 \mathrm{~h}$ in the autosampler (post-preparative stability), and below $-20^{\circ} \mathrm{C}$ (long-term stability). Moreover, six cycles of freeze-thaw stability and the storage stability of stock solutions were monitored. Stability monitoring was performed at two concentration levels (low and medium) in triplicates. Acceptable tolerances were $85-115 \%$ compared to the base level (time 0 ).

\subsection{Human Study}

The details of the study protocol for the controlled, single-center, and open-label clinical trial has been published previously [32]. All subjects gave their informed consent for inclusion before they participated in the study. The study was conducted in accordance with the Declaration of Helsinki, and the protocol was approved by the Ethics Committee of the Medical Association Hamburg. The study population covered exclusive users of five different nicotine-containing products (CC, EC, HTP, NRT, and OT) and a control group of non-users of any nicotine-containing product (NU). Each group consisted of 10 subjects. Complete urine voids were collected over three days of inpatient stay and pooled to yield $12 \mathrm{~h}$ urine samples $(12 \mathrm{~h}$ periods: from $6 \mathrm{p} . \mathrm{m}$. to $7 \mathrm{a} . \mathrm{m}$. and from $7 \mathrm{a} . \mathrm{m}$. to 6 p.m.). The analysis of 3-OH-BaP comprised the $12 \mathrm{~h}$ urine samples on the last day (Day 3, $\mathrm{U} 6$ and $\mathrm{U} 7+8$ ) of the inpatient stay, as these samples were collected on the third day of confinement under controlled conditions (diet control, habit control), which is the longest time period of control within this study. Main characteristics (user group, sex, age, BMI, and $24 \mathrm{~h}$ urine volume of Day 3 ) of the study population are summarized in Table 2.

For data evaluation, creatinine levels were additionally determined using the Jaffé method [34]. Product use status was verified by the determination of urinary nicotine and its ten metabolites (=total nicotine equivalents, TNE) using SPE (96-well plates) and LCMS/MS analysis (HILIC column) by modification of a previously published method [35] (Table S1).

\subsection{Data Evaluation and Statistics}

The statistical parameters were evaluated with Prism (GraphPad, Version 9.0.2, La Jolla, CA, USA). All 3-OH-BaP values below the LLOQ were set to LLOQ/2 (25 pg/L). The urinary 3-OH-BaP concentrations of $12 \mathrm{~h}$ urine samples were referred to pg 3-OH-BaP in $24 \mathrm{~h}(\mathrm{pg} / 24 \mathrm{~h})$, calculated as concentration $1 \times 12 \mathrm{~h}$-urine volume $1+$ concentration $2 \times 12 \mathrm{~h}$ urine volume 2 . In addition, analyte concentrations were normalized based on 
the creatinine concentrations and reported as pg/g creatinine (urinary 3-OH-BaP concentration in $\mathrm{pg} / \mathrm{L}$ divided by the respective creatinine concentration in $\mathrm{g} / \mathrm{L}$ ). Mean values, standard deviations, and median values were calculated for each user group. Statistical differences between the 3-OH-BaP concentration of smokers and the five other groups were determined by applying the non-parametric Mann-Whitney $U$ test $(p$-value $<0.05)$. Statistical differences between the main characteristics of the user groups were determined by applying the non-parametric, one-way ANOVA test (Kruskal-Wallis; $p$-value $<0.05$ ). Correlation of 3-OH-BaP levels of smokers and smoking-dose-related variables (number of cigarettes smoked per day (CPD) and TNE) were obtained by linear regression and evaluated by calculation of the Spearman correlation coefficient.

Table 2. Main characteristics of the study population.

\begin{tabular}{cccccccc}
\hline \multirow{2}{*}{ User Groups $^{\mathbf{1}}$} & \multirow{2}{*}{$\mathbf{N}(\mathbf{m} / \mathbf{f})$} & \multicolumn{2}{c}{ Age (Years) } & \multicolumn{2}{c}{ BMI } & \multicolumn{2}{c}{ 24 h Urine Volume (mL) } \\
\cline { 3 - 7 } & & Mean & \pm SD & Mean & \pm SD & Mean & \pm SD \\
\hline CC & $10(6 / 4)$ & 35.1 & \pm 9.1 & 26.0 & \pm 3.9 & 2891 & \pm 828 \\
HTP & $10(6 / 4)$ & 36.1 & \pm 12 & 25.5 & \pm 3.2 & 2685 & \pm 1300 \\
OT & $10(9 / 1)$ & 28.1 & \pm 8.2 & 25.9 & \pm 4.2 & 2638 & \pm 1290 \\
EC & $10(6 / 4)$ & 38.4 & \pm 14 & 23.5 & \pm 2.7 & 1627 & \pm 664 \\
NRT & $10(5 / 5)$ & 35.3 & \pm 15 & 25.5 & \pm 3.5 & 1602 & \pm 802 \\
NU & $10(6 / 4)$ & 32.9 & \pm 8.8 & 24.7 & \pm 3.2 & 2475 & \pm 936 \\
$\sum$ all & $60(38 / 22)$ & 34.3 & \pm 11 & 25.2 & \pm 3.4 & 2320 & \pm 1090 \\
\hline
\end{tabular}

${ }^{1}$ User groups: conventional cigarettes (CC), heated tobacco products (HTP), oral tobacco (OT), electronic cigarettes (EC), nicotine replacement therapy (NRT), and non-users (NU).

\section{Results}

\subsection{Performance of the Analytical Method}

An LC-MS/MS method published by Sarkar et al. [25] was used as a starting point, further optimized, and finally validated for the quantification of urinary 3-OH-BaP. Sample preparation included enzymatic hydrolysis with glucuronidase/arylsulfatase from Helix pomatia, SPE extraction, and derivatization of the hydroxyl group with FMPT (Figure 2). The extracts were then analyzed by LC-MS/MS.

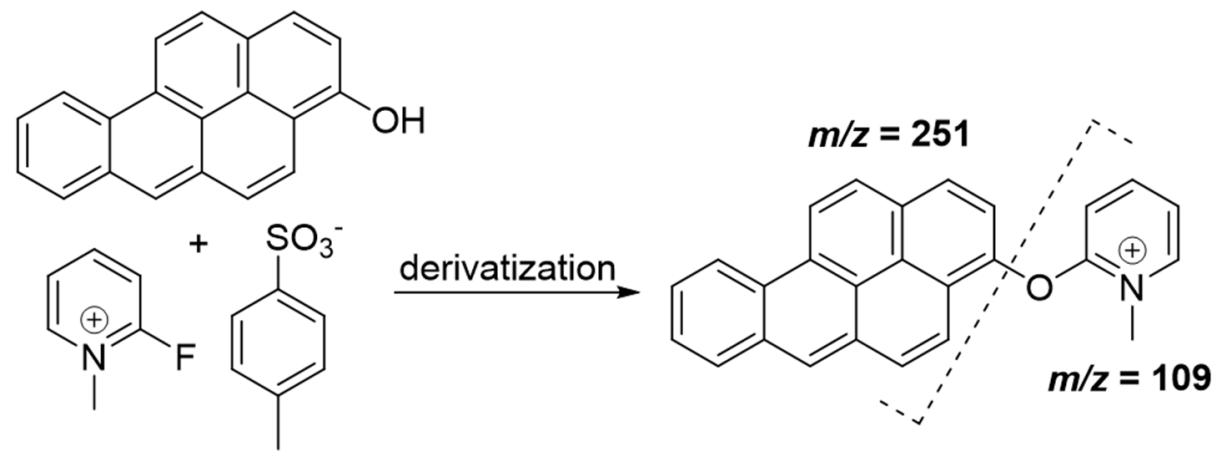

Figure 2. Derivatization of 3-OH-BaP with 2-fluoro-methylpyridinium-p-toluenesulfonate (FMPT).

The final method was validated according to FDA guidelines [33]. The method validation data are shown in Table 3. 
Table 3. Method validation data for the quantification of 3-OH-BaP in urine.

\begin{tabular}{|c|c|c|}
\hline Validation Parameter & Level & 3-OH-BaP \\
\hline LOD $^{1}$ & & $16.7 \mathrm{pg} / \mathrm{L}$ \\
\hline LLOQ & & $50 \mathrm{pg} / \mathrm{L}$ \\
\hline Calibration range & & $50-3221 \mathrm{pg} / \mathrm{L}$ \\
\hline \multicolumn{3}{|c|}{ Precision, intra-day, $\mathrm{N}=5$} \\
\hline & LLOQ: 50 pg/L & $10.1 \% \mathrm{CV}$ \\
\hline & Low: 100 pg/L & $12.0 \% \mathrm{CV}$ \\
\hline & Medium: $400 \mathrm{pg} / \mathrm{L}$ & $12.3 \% \mathrm{CV}$ \\
\hline & High: $1600 \mathrm{pg} / \mathrm{L}$ & $3.3 \% \mathrm{CV}$ \\
\hline \multicolumn{3}{|c|}{ Precision, inter-day, $\mathrm{N}=3 \times 5$} \\
\hline & LLOQ: 50 pg/L & $7.9 \% \mathrm{CV}$ \\
\hline & Low: $100 \mathrm{pg} / \mathrm{L}$ & $9.0 \% \mathrm{CV}$ \\
\hline & Medium: $400 \mathrm{pg} / \mathrm{L}$ & $8.0 \% \mathrm{CV}$ \\
\hline & High: $1600 \mathrm{pg} / \mathrm{L}$ & $5.8 \% \mathrm{CV}$ \\
\hline \multicolumn{3}{|c|}{ Accuracy, intra-day, $\mathrm{N}=5$} \\
\hline & LLOQ: 50 pg/L & $101.8 \%$ \\
\hline & Low: $100 \mathrm{pg} / \mathrm{L}$ & $105.1 \%$ \\
\hline & Medium: $400 \mathrm{pg} / \mathrm{L}$ & $94.0 \%$ \\
\hline & High: $1600 \mathrm{pg} / \mathrm{L}$ & $98.2 \%$ \\
\hline \multicolumn{3}{|c|}{ Accuracy, inter-day, $\mathrm{N}=3 \times 5$} \\
\hline & LLOQ: 50 pg/L & $105.8 \%$ \\
\hline & Low: $100 \mathrm{pg} / \mathrm{L}$ & $110.7 \%$ \\
\hline & Medium: $400 \mathrm{pg} / \mathrm{L}$ & $95.6 \%$ \\
\hline & High: 1600 pg/L & $99.6 \%$ \\
\hline \multicolumn{3}{|l|}{ Recovery ${ }^{2,3}, \mathrm{~N}=6$} \\
\hline & Low: 200 pg/L & $121.3 \%$ \\
\hline & Medium: $640 \mathrm{pg} / \mathrm{L}$ & $108.9 \%$ \\
\hline & High: $1600 \mathrm{pg} / \mathrm{L}$ & $89.1 \%$ \\
\hline \multicolumn{3}{|l|}{ Matrix effect ${ }^{3}, N=3$} \\
\hline & Low: 200 pg/L & $+31.4 \%$ \\
\hline & High: $1600 \mathrm{pg} / \mathrm{L}$ & $+43.3 \%$ \\
\hline & Low: IS & $+25.3 \%$ \\
\hline & High: IS & $+47.9 \%$ \\
\hline \multicolumn{3}{|l|}{ Re-injection ${ }^{3}, \mathrm{~N}=3 \times 3$} \\
\hline & Low: 200 pg/L & $5.0 \% \mathrm{CV}$ \\
\hline & Medium: $640 \mathrm{pg} / \mathrm{L}$ & $4.6 \% \mathrm{CV}$ \\
\hline
\end{tabular}

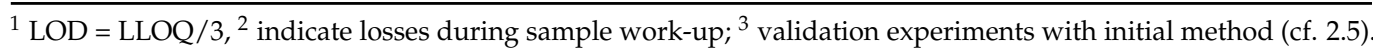

The selectivity was proven by analyzing six different analyte-free urine samples. No interfering signals at the same retention times as the analyte or internal standard MRM transitions were detected. Spiking the six samples with the analyte resulted in a mean accuracy of $86.4 \%$.

The precision was evaluated by calculation of the relative standard deviation expressed as CVs, which should not exceed $15 \% \mathrm{CV}(20 \% \mathrm{CV}$ at LLOQ). Intra-day precision ranged from $3.3 \%$ to $12.3 \%$ for the different concentration levels. The CVs for the inter-day precision were between $5.8 \%$ and $9.0 \%$. The determined intra-day accuracy rates for the LLOQ $(101.8 \%)$, the low $(105.1 \%)$, the medium $(94.0 \%)$, and the high $(98.2 \%)$ concentration level were within the acceptable range. Inter-day accuracy was also within the range.

For the quantification method, an LLOQ of $50 \mathrm{pg} / \mathrm{L}$ was confirmed by the analysis of five independent spiked urine samples on three consecutive days. The LOD was defined as LLOQ/3 and amounted to $16.7 \mathrm{pg} / \mathrm{L}$, showing a signal-to-noise ratio of approximately five. A linear response was found for the calibration range of 50-3221 pg/L.

High recovery rates ( 89 to $121 \%$ ) were obtained despite numerous steps in the sample work-up, including enzymatic hydrolysis, SPE extraction, and derivatization. A positive $\mathrm{ME}$ in the urine of $+31 \%$ to $+43 \%$ was observed for the derivate of $3-\mathrm{OH}-\mathrm{BaP}$. The MEs were fully compensated by the IS. There was no significant carryover, evaluated by a blank sample injected after five consecutive injections of samples with high concentrations. The 
post-preparative stability of the final extracts was proven in the autosampler at $10{ }^{\circ} \mathrm{C}$ for at least $72 \mathrm{~h}$. The reproducibility of re-injection was analyzed by measuring samples with low and medium analyte concentrations in triplicates at three different time points, resulting in CVs of $5.0 \%$ and $4.6 \%$, respectively.

Urine samples spiked at two concentration levels with free 3-OH-BaP were stored below $-20{ }^{\circ} \mathrm{C}$ and analyzed after 1,3 , and 7 days. The accuracy decreased gradually from $106 \%$ and $74 \%$ on day 1 to $93 \%$ and $58 \%$ on day 7 for $200 \mathrm{pg} / \mathrm{L}$ and $640 \mathrm{pg} / \mathrm{L}$, respectively. Apparently, free 3-OH-BaP was not stable in urine. Degradation was also observed for standard solutions of the analyte and the internal standard in their free form, and thus, fresh solutions needed to be prepared on the day of use. As an alternative for the less stable free 3-OH-BaP, the stability of the glucuronide (3-OH-BaP-Gluc) was investigated as well. 3-OH-BaP-Gluc proved to be stable in urine for at least $30 \mathrm{~h}$ at room temperature (short-term stability) and for at least 15 months when stored below $-20{ }^{\circ} \mathrm{C}$ (long-term stability). The analyte in its conjugated form was stable through six freeze/thaw cycles in urine samples stored below $-20^{\circ} \mathrm{C}$. The stock solution of the 3-OH-BaP-Gluc in water (c $=50 \mu \mathrm{g} / \mathrm{mL}$ ) was stable for 3.3 years when stored below $-20{ }^{\circ} \mathrm{C}$. Consequently, 3$\mathrm{OH}-\mathrm{BaP}-\mathrm{Gluc}$ was established for the preparation of QC material and for calibration. In analogy, the glucuronide ${ }^{13} \mathrm{C}_{6}-3-\mathrm{OH}-\mathrm{BaP}-\mathrm{Gluc}$ was used as an internal standard. Since no interferences were found in the MRM transition, ${ }^{13} \mathrm{C}_{6}-3-\mathrm{OH}-\mathrm{BaP}-\mathrm{Gluc}$ was established as IS in the final method to compensate losses during sample work-up. Exemplary MRM chromatograms of non-smoker urine, a QC sample at low concentration, and a smoker urine sample are illustrated in Figure 3.

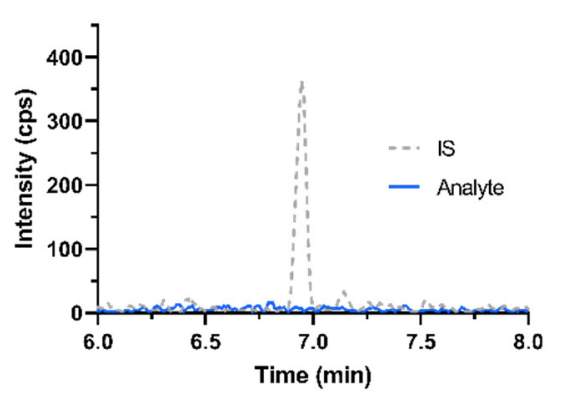

(a)

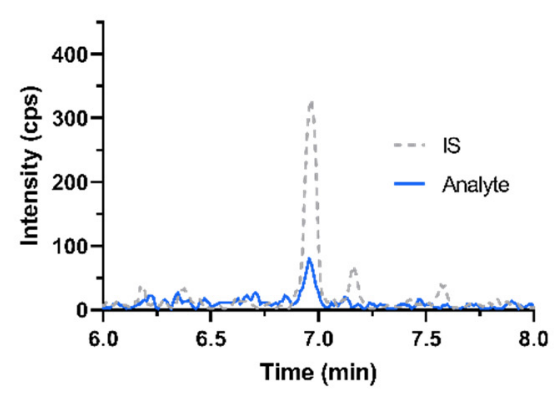

(b)

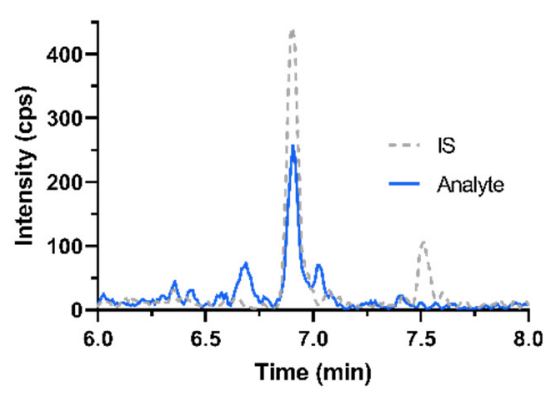

(c)

Figure 3. MRM chromatograms of the analyte 3-OH-BaP $(m / z 360 \rightarrow 251)$ and the internal standard ${ }^{13} \mathrm{C}_{6}-3-\mathrm{OH}-\mathrm{BaP}$ $(\mathrm{m} / \mathrm{z} 366 \rightarrow 257)$. (a): Non-smoker urine sample (<LOD); (b): quality control sample with low concentration $(\mathrm{c}=162 \mathrm{pg} / \mathrm{L})$; (c): smoker urine sample (c $=470 \mathrm{pg} / \mathrm{L})$.

\subsection{Human Study-Urinary Excretion of 3-OH-BaP}

The validated method was applied to urine samples from a controlled clinical study [32]. Each group consisted of 10 subjects, resulting in a total number of 60 subjects stratified by product use. The study population was assigned to one of the five groups of users of different nicotine-containing products (CC, EC, HTP, NRT, and OT) based on their product use or to the control group of NU. The main characteristics of the subjects are summarized in Table 2. The confined and diet-controlled clinical study was chosen to ascertain similar (low) exposure to BaP from sources other than product use such as diet or ambient air. Therefore, 3-OH-BaP was quantitated in the $12 \mathrm{~h}$ urine samples of the last study day, as this was the longest time period under controlled conditions. Group comparisons were performed based on the total amount of urinary 3-OH-BaP excreted over $24 \mathrm{~h}(\mathrm{pg} / 24 \mathrm{~h})$, as summarized in Figure 4 and Table 4. 


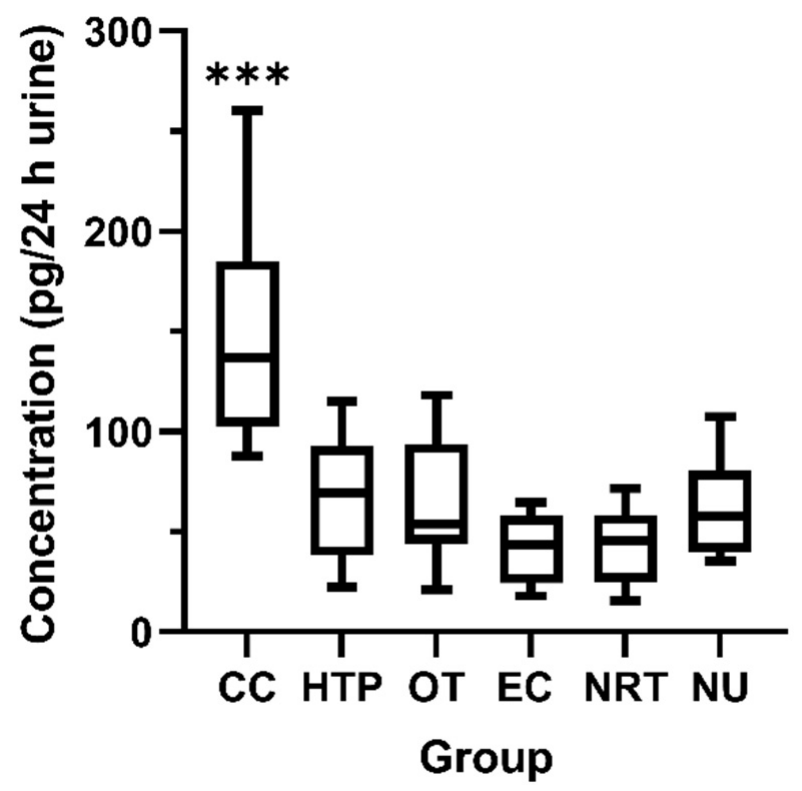

Figure 4. Box plots for urinary 3-OH-BaP excretion (pg/24 h) of six different user groups on Day 3. Boxes and lines represent the range of twenty-fifth/seventy-fifth percentile and the median value. The whiskers illustrate the minimum and maximum concentration. Differences between the smoking (CC) and all other groups were found to be significant when using the non-parametric Mann-Whitney $U$ test ${ }^{* * *}: p$-value $\left.<0.002\right)$.

Table 4. Descriptive statistics for urinary 3-OH-BaP excretion (pg/24 h) of six different user groups.

\begin{tabular}{|c|c|c|c|c|c|c|}
\hline & \multicolumn{6}{|c|}{ 3-OH-BaP $(\mathrm{pg} / 24 \mathrm{~h})^{1}$} \\
\hline & $\mathrm{CC}$ & HTP & OT & EC & NRT & NU \\
\hline Mean \pm SD & $149.0 \pm 57.0$ & $67.14 \pm 32.6$ & $65.96 \pm 32.3$ & $40.68 \pm 16.6$ & $43.31 \pm 19.2$ & $61.88 \pm 23.4$ \\
\hline Median & 136.9 & 69.40 & 53.75 & 43.35 & 45.40 & 57.60 \\
\hline Min-max & $87.70-260.3$ & $22.30-115.1$ & 21.10-118.0 & $17.90-64.60$ & $15.30-71.50$ & $35.40-107.3$ \\
\hline$<$ LLOQ, N (\%) ${ }^{2}$ & $8(40 \%)$ & $20(100 \%)$ & $20(100 \%)$ & $20(100 \%)$ & $19(95 \%)$ & $20(100 \%)$ \\
\hline
\end{tabular}

${ }^{1}$ Levels of 3-OH-BaP excreted within $24 \mathrm{~h}(\mathrm{~N}=10$ per group). Concentrations $<$ LLOQ were set to LLOQ/2 (25 pg/L) for calculation of $12 \mathrm{~h}$ and $24 \mathrm{~h}$ excretion; ${ }^{2}$ referred to the concentration in $\mathrm{pg} / \mathrm{L}$ of $12 \mathrm{~h}$ urine samples $(\mathrm{N}=20$ per group).

The highest mean concentration of $149 \mathrm{pg} / 24 \mathrm{~h}$ was determined for smokers, with $60 \%$ of samples above the LLOQ in this group. In contrast, all samples from the other groups, including the NU, were not quantifiable, except for one $12 \mathrm{~h}$ urine sample in the NRT group. Mean values varied between groups due to differences in $12 \mathrm{~h}$ urine volumes (cf. Table 1). Urinary 3-OH-BaP excretion was significantly higher $(p$-value $<0.002)$ in smokers compared to all other groups (Figure 4).

\subsection{Correlation of 3-OH-BaP with Smoking Specific Parameters}

To investigate the specificity of 3-OH-BaP as biomarker of tobacco smoke exposure, urinary 3-OH-BaP levels were plotted against the smoking dose, as indicated by the number of cigarettes smoked per day (CPD) (Figure 5a) and the total nicotine equivalents (TNE) excreted in urine (Figure $5 b$ ). Linear regression showed a moderate correlation (of borderline significance) between the 3-OH-BaP concentrations and CPD (Spearman's $r=0.63$ ) and a weak (statistically not significant) correlation between $3-\mathrm{OH}-\mathrm{BaP}$ concentrations and urinary TNE (Spearman's $r=0.52$ ). A reason for the only moderate or weak correlation could be the relatively small sample number. Nevertheless, the positive correlation of 3$\mathrm{OH}-\mathrm{BaP}$ with these smoking dose parameters indicates that urinary $3-\mathrm{OH}-\mathrm{BaP}$ is a suitable biomarker to assess $\mathrm{BaP}$ exposure by cigarette smoking. 
(a)

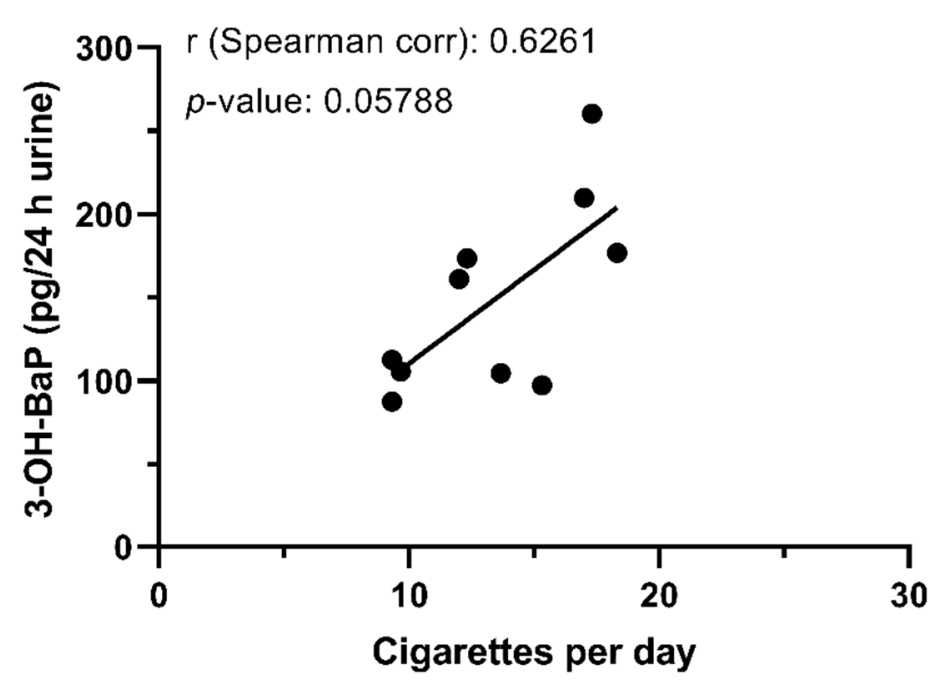

(b)

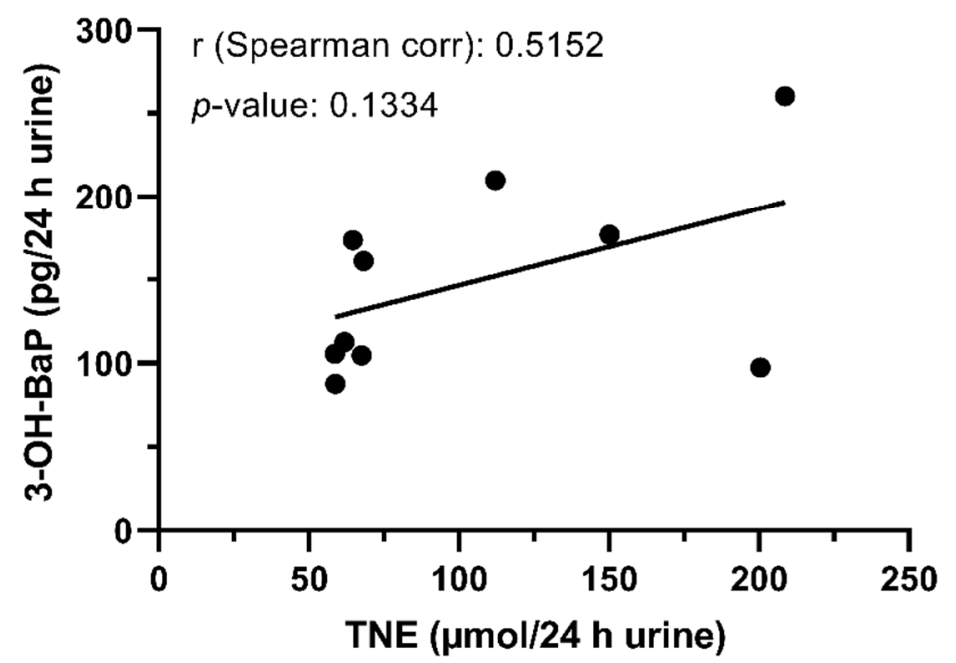

Figure 5. Spearman's correlation between urinary 3-OH-BaP concentrations and (a) number of cigarettes smoked per day (CPD) and (b) urinary total nicotine equivalents (TNE) of CC users on Day $3(\mathrm{~N}=10)$.

\section{Discussion}

\subsection{Analytical Method}

BaP exposure is most frequently investigated by analyzing the urinary metabolite 3$\mathrm{OH}-\mathrm{BaP}$. Numerous methods have been reported for the quantification of this biomarker in occupationally exposed workers [19-22]. However, these methods generally lack sensitivity for the quantification of 3-OH-BaP in the non-occupationally exposed population. The purpose of the current work was to develop and validate a sensitive method for the quantification of trace amounts of urinary 3-OH-BaP in cohorts of non-occupationally exposed subjects, i.e., in clinical and epidemiological studies. The procedure described by Sarkar et al. [25] was used as a starting point and further modified with respect to the extraction procedure and use of conjugated standards in order to achieve the required sensitivity along with a sufficient sample throughput and robustness. One important improvement in our method in terms of repeatability and accuracy was the implementation of ${ }^{13} \mathrm{C}_{6}-3-\mathrm{OH}-\mathrm{BaP}-\mathrm{Gluc}$ as an internal standard. Using the glucuronides as standard and IS material proved to be superior to the unstable free analyte during method validation, calibration, and quality control procedures. In native urine samples, 3-OH-BaP was found to be present in its conjugated form in urine at almost 100\% [18]; thus, instability of the 
analyte would not present an issue in real samples. Another advantage would be that ${ }^{13} \mathrm{C}_{6}-3-\mathrm{OH}-\mathrm{BaP}-\mathrm{Gluc}$ could also compensate for losses during enzymatic hydrolysis.

Thus far, only a few methods have been described in the literature that are capable of quantifying BaP exposures in the low pg/L range besides Sarkar et al. [25] and our method. One other method has been published with a similar LLOQ of $50 \mathrm{pg} / \mathrm{L}$ by application of liquid chromatography-fluorescence detection (FD) and automated off-line solid-phase extraction [28,36]. While fluorescence detection achieved comparable sensitivity, our method is more specific and selective, applying MRM detection of several analyte-specific mass transitions. Further, LC-MS/MS methods were developed for the quantification of 3-OH-BaP, yielding higher LLOQs. Simon et al. published an automated column-switching high-performance liquid chromatography method for the determination of 3-OH-BaP in urine, yielding quantification limits of approximately $400 \mathrm{pg} / \mathrm{L}[22,29,30,37]$. Several groups analyzed 3-OH-BaP by employing derivatization of the hydroxyl group with dansyl chloride and subsequent analysis by LC-MS/MS, resulting in LLOQs of $250 \mathrm{pg} / \mathrm{L}$ [26], $300 \mathrm{pg} / \mathrm{L}$ [24], and $580 \mathrm{pg} / \mathrm{L}$ [27]. Richter-Brockmann et al. followed a different approach by means of GC-MS using atmospheric pressure laser ionization (APLI). A higher sensitivity compared to our method was reported by the use of APLI, which apparently improved the ionization yields for the methyl ether of 3-OH-BaP with an LLOQ of $1.8 \mathrm{pg} / \mathrm{L}$ [31]. Additionally, ascorbic acid was added as an antioxidant before enzymatic cleavage to protect the resulting free 3-OH-BaP from oxidative decomposition during the following work-up and analysis-a procedure that has been established for other PAHs before [38]. Hence, we investigated the addition of ascorbic acid in our method as well. QC samples were analyzed with and without the addition of ascorbic acid, showing no differences in terms of accuracy, sensitivity, and specificity (data not shown). Since the overall variability appeared to be slightly improved by the addition of ascorbic acid, this procedure was implemented into the final method for validation of accuracy, precision, calibration range, and LLOQ and for analysis of the clinical study samples.

\subsection{Human Study}

The validated method was applied to urine samples of NUs and users of five different nicotine-containing products (CC, EC, NRT, HTP, and OT) who participated in a controlled clinical trial [32]. The aim was not only to investigate whether cigarette smokers and non-smokers differ but also whether cigarette smokers differ from other users of potentially reduced-risk products in terms of exposure to various toxicants, among them BaP. No significant differences were found in terms of the general study group characteristics for age, BMI, and urine volume between the different groups.

The group of CC smokers and NU differ significantly in terms of urinary excretion of 3-OH-BaP. With mean values of $149.0 \mathrm{pg} / 24 \mathrm{~h}$ urine ( $225.9 \mathrm{pg} / \mathrm{g}$ creatinine, Table S2) and $61.88 \mathrm{pg} / 24 \mathrm{~h}$ urine $(90.73 \mathrm{pg} / \mathrm{g}$ creatinine, Table S2) in CC smokers and NUs, respectively, the determined values were in the same range as reported in the literature $[25,28,30]$.

Barbeau et al. [28] analyzed urinary 3-OH-BaP in non-occupationally exposed nonsmokers and smokers. They found an average concentration of $0.009 \mathrm{nmol} / \mathrm{mol}$ creatinine for non-smokers and $0.023 \mathrm{nmol} / \mathrm{mol}$ creatinine for smokers. These concentrations equaled $45 \mathrm{pg} / 24 \mathrm{~h}$ urine and $155 \mathrm{pg} / 24 \mathrm{~h}$ urine, respectively, assuming a mean $24 \mathrm{~h}$ urine volume of $1.5 \mathrm{~L}$ and a mean urinary creatinine concentration of $1.5 \mathrm{~g} / \mathrm{L}$ [28]. The mean concentrations for non-smokers and smokers of $59 \mathrm{pg} / 24 \mathrm{~h}$ urine and $131 \mathrm{pg} / 24 \mathrm{~h}$ urine determined by Lafontaine et al. [30] were in the same range. Sarkar et al. [25] showed, through the analysis of various smoking-specific biomarkers, including 3-OH-BaP, a significant reduction in biomarker concentrations in the group that stopped consuming conventional cigarettes after the baseline of the study. The values at post-baseline were $155 \mathrm{pg} / 24 \mathrm{~h}$ urine for the continuous smoking group and $56 \mathrm{pg} / 24 \mathrm{~h}$ urine for the group that had completely quit using any tobacco product for eight days, which was comparable to non-smokers. In other recent studies analyzing 3-OH-BaP in the urine of non-smokers and smokers, higher concentrations were found in both groups. Concentrations in non-occupationally 
subjects $(\mathrm{N}=4-7)$ were found in a range of $<\mathrm{LOD}$ to $820 \mathrm{pg} / \mathrm{L}$ for non-smokers and of 320 to $2150 \mathrm{pg} / \mathrm{L}$ for smokers [26,27,31]. Richter-Brockmann et al. speculated that the addition of ascorbic acid may have led to higher concentrations due to improved stability of the analyte. However, our studies using QCs could not prove this hypothesis since comparable concentrations of 3-OH-BaP were observed for identical samples worked-up with and without the addition of ascorbic acid.

In contrast to most studies that investigated exposure in smokers and non-smokers only, Sarkar et al. studied 3-OH-BaP in subjects switching from CC to a snus product. They observed a reduction of about $45 \%$ in smokers eight days after switching to snus use, which was in the range of the cessation arm in this study, with a decrease of about 56\% [25]. To the best of our knowledge, our clinical study was the first to analyze urinary 3-OH$\mathrm{BaP}$ concentrations to distinguish between smokers and non-smokers, and additionally included four other groups of users of new generation nicotine/tobacco products, such as HTP, OT, EC, and NRT. The four other nicotine user groups could be clearly distinguished from the smokers in terms of their urinary 3-OH-BaP levels, which were indistinguishable from those of NU (99\% < LLOQ). The somewhat lower concentrations of EC and NRT users can be explained by the normalization with the $24 \mathrm{~h}$ urine volume. It is important to emphasize that the urine volumes collected did not differ significantly between the different user groups (Table 2).

In addition to the number of CPD, urinary cotinine concentration or TNE are commonly used as a biomarker of exposure to nicotine products and have been used for the classification of smoking status. Richter-Brockmann et al. showed a positive correlation of urinary 3-OH-BaP concentration to CPD and cotinine, respectively $\left(\mathrm{R}^{2}=0.88\right.$ each) [31]. We could confirm the positive correlation between urinary 3-OH-BaP and smoking dose, measured as CPD or TNE in our study (Figure 5).

This study was performed under confined and diet-controlled conditions to reduce the influence of other sources for BaP exposure and to also ascertain compliance of single product use during the inpatient stay. In the clinical study, urine voids were collected over three days, which is regarded as a sufficient time period for the washout of 3-OH-BaP. This was evident, for example, when looking at the progression from Day 1 to Day 3 of a non-compliant NRT user (Figure 6), as identified by the observed CEMA (N-acetyl-S-(2cyanoethyl)-L-cysteine) concentration (a biomarker of exposure to acrylonitrile), although reporting exclusive NRT use for the last three months. Smoking was identified as a major source of acrylonitrile exposure in several studies [39-41]. A CEMA cut-off between 0.4 and $0.7 \mu \mathrm{g} / \mathrm{L}$ was recently suggested [42]. The non-compliant subject in the NRT user group showed a CEMA concentration of $86 \mu \mathrm{g} / \mathrm{L}$ on Day 1 (U0) equal to $84 \mu \mathrm{g} / 12 \mathrm{~h}$ urine fraction, strongly indicating cigarette smoking before the study started.

The high 3-OH-BaP concentration ( $>200 \mathrm{pg} / 12 \mathrm{~h}$ urine) in the U0 fraction supports these findings. A downward trend was observed from Day 1 to Day 3, indicating that non-compliant behavior and other sources of $\mathrm{BaP}$ exposure could be excluded during the course of the study. Despite possible non-compliance and other BaP exposure sources, NUs and all other nicotine user groups were significantly distinguishable from CC smokers in the urine samples collected before the study started (U0) ( $p$-value $<0.05$, Table S3). These findings show that 3-OH-BaP is significantly elevated in smokers in an uncontrolled setting as well, emphasizing the suitability of $3-\mathrm{OH}-\mathrm{BaP}$ as a biomarker to discriminate cigarette smoking from other nicotine-containing products such as e-cigarettes, smokeless and oral tobacco, or heated tobacco products. Since only a small sample size of each user group $(\mathrm{N}=10)$ was used here, these findings would need to be confirmed in larger cohorts. 


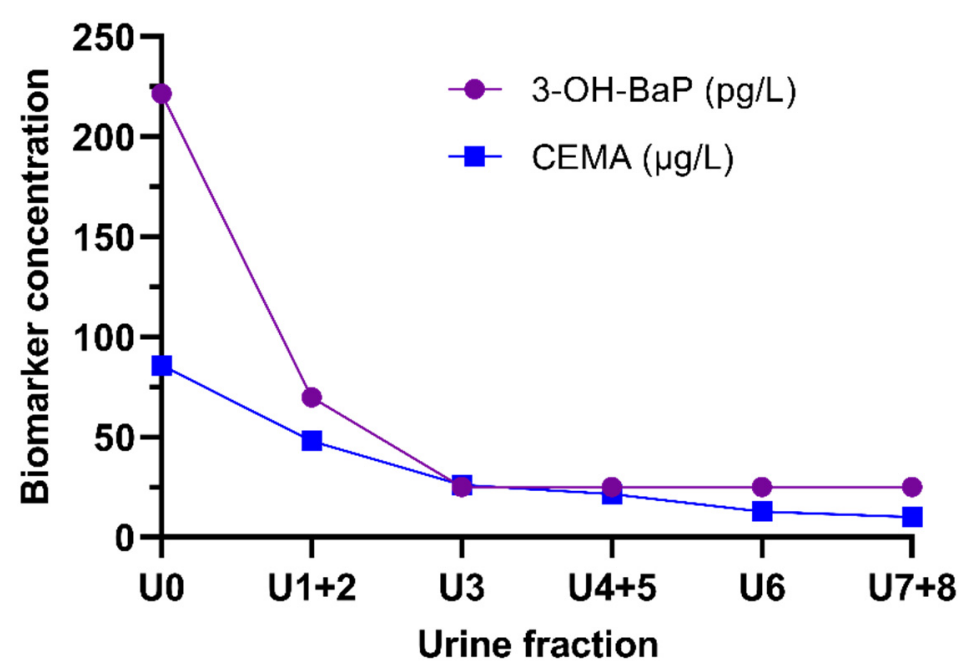

Figure 6. Progression of 3-OH-BaP $(\mathrm{pg} / \mathrm{L})$ and CEMA $(\mu \mathrm{g} / \mathrm{L})$ concentration of an NRT user over the three study days.

\section{Conclusions}

The new LC-MS/MS method is highly sensitive and allows for quantification of urinary 3-OH-BaP in cohorts of non-occupationally exposed subjects due to high throughput. Covering a broad, linear calibration range and an LLOQ of $50 \mathrm{pg} / \mathrm{L}$, the actual method is suitable for the quantification of occupationally and non-occupationally exposed populations. Smokers can be differentiated from non-smokers as well as from users of new generation tobacco/nicotine and oral tobacco products. A moderate correlation between urinary $3-\mathrm{OH}-\mathrm{BaP}$ and the smoking dose was observed. Hence, $3-\mathrm{OH}-\mathrm{BaP}$ is a suitable biomarker to discriminate smokers from users of potentially reduced-risk products. The method is also suitable for assessing low exposures to $\mathrm{BaP}$ originating from diet and ambient air.

Supplementary Materials: The following are available online at https://www.mdpi.com/article/10 .3390 / separations8100171/s1, Table S1: Descriptive statistics for TNE ( $\mu \mathrm{mol} / 24 \mathrm{~h}$ urine) of six different user groups on Day 3, Table S2: Descriptive statistics for urinary 3-OH-BaP excretion (pg/g creatinine) of six different user groups on Day 3, Table S3: Descriptive statistics for urinary 3-OH-BaP excretion (pg/12 h urine) of six different user groups on Day-1.

Author Contributions: Conceptualization, M.S., G.S. and N.P.; methodology, N.R. and H.-W.H.; validation, N.R. and H.-W.H.; formal analysis, N.R.; investigation, N.R.; writing-original draft preparation, N.R.; writing-review and editing, M.S., G.S. and N.P.; supervision, N.P.; project administration, N.P.; funding acquisition, M.S. All authors have read and agreed to the published version of the manuscript.

Funding: This study was funded with a grant from the Foundation for a Smoke-Free World, Inc. ("FSFW"), a US nonprofit 501 (c) (3) private foundation. This study is, under the terms of the grant agreement with FSFW, editorially independent of FSFW. The contents, selection, and presentation of facts, as well as any opinions expressed herein, are the sole responsibility of the authors and under no circumstances shall be regarded as reflecting the positions of the FSFW. The FSFW accepts charitable gifts from PMI Global Services Inc. (PMI); under FSFW's Bylaws and Pledge Agreement with PMI, the FSFW is independent of PMI and the tobacco industry.

Institutional Review Board Statement: The study was conducted according to the guidelines of the Declaration of Helsinki, and the protocol was approved by the Ethics Committee of the Medical Association Hamburg (reference number: PV7084, date of approval: 10 September 2019).

Informed Consent Statement: Informed consent was obtained from all subjects involved in the study. 
Data Availability Statement: The data presented here are available on request from the corresponding author.

Acknowledgments: The authors thank CTC North GmbH (Hamburg, Germany) for conducting the clinical study, and our colleagues from ABF for their assistance in organizing and managing the clinical study, and for analysis of the urinary nicotine metabolites.

Conflicts of Interest: The authors declare no conflict of interest. The funders had no role in the design of the study; in the collection, analyses, or interpretation of data; in the writing of the manuscript, or in the decision to publish the results.

\section{References}

1. Lijinsky, W. The formation and occurrence of polynuclear aromatic hydrocarbons associated with food. Mutat. Res. Genet. Toxicol. 1991, 259, 251-261. [CrossRef]

2. Hattemer-Frey, H.A.; Travis, C.C. Benzo-a-pyrene: Environmental partitioning and human exposure. Toxicol. Ind. Health 1991, 7, 141-157. [CrossRef] [PubMed]

3. IARC. Monographs on the Evaluation of Carcinogenic Risks to Humans. Some Non-Heterocyclic Polycyclic Aromatic Hydrocarbons and Some Related Exposures; IRAC: Lyon, France, 2010; Volume 92.

4. Rodgman, A.; Perfetti, T. The Composition of Cigarette Smoke: A Catalogue of the Polycyclic Aromatic Hydrocarbons. Contrib. Tob. Res. 2006, 22, 13-69. [CrossRef]

5. IARC. Monographs on the Evaluation of Carcinogenic Risks to Humans. Polynuclear Aromatic Compounds, Part 1: Chemical, Environmental and Experimental Data; IRAC: Lyon, France, 1983; Volume 32.

6. IARC. Monographs on the Evaluation of Carcinogenic Risks to Humans. Chemical Agents and Related Occupations; IRAC: Lyon, France, 2012; Volume $100 \mathrm{~F}$.

7. Scherer, G.; Frank, S.; Riedel, K.; Meger-Kossien, I.; Renner, T. Biomonitoring of Exposure to Polycyclic Aromatic Hydrocarbons of Nonoccupationally Exposed Persons. Cancer Epidemiol. Biomark. Prev. 2000, 9, 373-380.

8. Jacob, J.; Seidel, A. Biomonitoring of polycyclic aromatic hydrocarbons in human urine. J. Chromatogr. B: Anal. Technol. Biomed. Life Sci. 2002, 778, 31-47. [CrossRef]

9. Ramsauer, B.; Sterz, K.; Hagedorn, H.W.; Engl, J.; Scherer, G.; McEwan, M.; Errington, G.; Shepperd, J.; Cheung, F. A liquid chromatography/tandem mass spectrometry (LC-MS/MS) method for the determination of phenolic polycyclic aromatic hydrocarbons (OH-PAH) in urine of non-smokers and smokers. Anal. Bioanal. Chem. 2011, 399, 877-889. [CrossRef] [PubMed]

10. Dor, F.; Dab, W.; Empereur-Bissonnet, P.; Zmirou, D. Validity of biomarkers in environmental health studies: The case of PAHs and benzene. Crit. Rev. Toxicol. 1999, 29, 129-168. [CrossRef]

11. Deutsche Forschungsgemeinschaft (DFG). Benzo[a]pyrene. In MAK Collection: Occupational toxicants, Part 1; Commission, M., Ed.; Wiley-VCH Verlag: Heidelberg, Germany, 2012; Volume 27.

12. Conney, A.H.; Chang, R.L.; Jerina, D.M.; Caroline Wei, S.J. Studies on the Metabolism of Benzo[a]Pyrene and Dose-Dependent Differences in the Mutagenic Profile of Its Ultimate Carcinogenic Metabolite. Drug Metab. Rev. 1994, 26, 125-163. [CrossRef]

13. Gelboin, H.V. Benzo[a]pyrene metabolism, activation and carcinogenesis: Role and regulation of mixed-function oxidases and related enzymes. Physiol. Rev. 1980, 60, 1107-1166. [CrossRef]

14. Andreas, L.; William, M.B. Metabolic Activation and Detoxification of Polycyclic Aromatic Hydrocarbons. In The Carcinogenic Effects of Polycyclic Aromatic Hydrocarbons; World Scientific: London, UK, 2005; pp. 19-96.

15. Verma, N.; Pink, M.; Rettenmeier, A.W.; Schmitz-Spanke, S. Review on proteomic analyses of benzo[a]pyrene toxicity. Proteomics 2012, 12, 1731-1755. [CrossRef]

16. Zhong, Y.; Carmella, S.G.; Hochalter, J.B.; Balbo, S.; Hecht, S.S. Analysis of r-7,t-8,9,c-10-Tetrahydroxy-7,8,9,10-tetrahydrobenzo[a] pyrene in Human Urine: A Biomarker for Directly Assessing Carcinogenic Polycyclic Aromatic Hydrocarbon Exposure Plus Metabolic Activation. Chem. Res. Toxicol. 2011, 24, 73-80. [CrossRef] [PubMed]

17. Richter-Brockmann, S.; Dettbarn, G.; Jessel, S.; John, A.; Seidel, A.; Achten, C. GC-APLI-MS as a powerful tool for the analysis of BaP-tetraol in human urine. J. Chromatogr. B 2018, 1100-1101, 1-5. [CrossRef] [PubMed]

18. Luo, K.; Gao, Q.; Hu, J. Determination of 3-Hydroxybenzo[a]pyrene Glucuronide/Sulfate Conjugates in Human Urine and Their Association with 8-Hydroxydeoxyguanosine. Chem. Res. Toxicol. 2019, 32, 1367-1373. [CrossRef]

19. Gündel, J.; Angerer, J. High-performance liquid chromatographic method with fluorescence detection for the determination of 3-hydroxybenzo[a]pyrene and 3-hydroxybenz[a]anthracene in the urine of polycyclic aromatic hydrocarbon-exposed workers. J. Chromatogr. B Biomed. Sci. Appl. 2000, 738, 47-55. [CrossRef]

20. Gündel, J.; Schaller, K.H.; Angerer, J. Occupational exposure to polycyclic aromatic hydrocarbons in a fireproof stone producing plant: Biological monitoring of 1-hydroxypyrene, 1-, 2-, 3- and 4-hydroxyphenanthrene, 3-hydroxybenz(a)anthracene and 3-hydroxybenzo(a)pyrene. Int. Arch. Occup. Environ. Health 2000, 73, 270-274. [CrossRef]

21. Raponi, F.; Bauleo, L.; Ancona, C.; Forastiere, F.; Paci, E.; Pigini, D.; Tranfo, G. Quantification of 1-hydroxypyrene, 1- and 2-hydroxynaphthalene, 3-hydroxybenzo[a]pyrene and 6-hydroxynitropyrene by HPLC-MS/MS in human urine as exposure biomarkers for environmental and occupational surveys. Biomarkers 2017, 22, 575-583. [CrossRef] 
22. Förster, K.; Preuss, R.; Rossbach, B.; Bruning, T.; Angerer, J.; Simon, P. 3-Hydroxybenzo[a]pyrene in the urine of workers with occupational exposure to polycyclic aromatic hydrocarbons in different industries. Occup. Environ. Med. 2008, 65, 224-229. [CrossRef]

23. Gendre, C.; Lafontaine, M.; Morele, Y.; Payan, J.-P.; Simon, P. Relationship Between Urinary Levels of 1-Hydroxypyrene and 3-Hydroxybenzo[a]pyrene for Workers Exposed to Polycyclic Aromatic Hydrocarbons. Polycyclic Aromat. Compd. 2002, 22, 761-769. [CrossRef]

24. Luo, K.; Gao, Q.; Hu, J. Derivatization method for sensitive determination of 3-hydroxybenzo [a] pyrene in human urine by liquid chromatography-electrospray tandem mass spectrometry. J. Chromatogr. A 2015, 1379, 51-55. [CrossRef]

25. Sarkar, M.; Liu, J.; Koval, T.; Wang, J.; Feng, S.; Serafin, R.; Jin, Y.; Xie, Y.; Newland, K.; Roethig, H.J. Evaluation of biomarkers of exposure in adult cigarette smokers using Marlboro Snus. Nicotine Tob. Res. 2010, 12, 105-116. [CrossRef] [PubMed]

26. Yao, L.; Yang, J.; Liu, B.; Zheng, S.; Wang, W.; Zhu, X.; Qian, X. Development of a sensitive method for the quantification of urinary 3-hydroxybenzo[a]pyrene by solid phase extraction, dansyl chloride derivatization and liquid chromatography-tandem mass spectrometry detection. Anal. Methods 2014, 6, 6488-6493. [CrossRef]

27. Hu, H.; Liu, B.; Yang, J.; Lin, Z.; Gan, W. Sensitive determination of trace urinary 3-hydroxybenzo[a]pyrene using ionic liquidsbased dispersive liquid-liquid microextraction followed by chemical derivatization and high performance liquid chromatographyhigh resolution tandem mass spectrometry. J. Chromatogr. B 2016, 1027, 200-206. [CrossRef]

28. Barbeau, D.; Maître, A.; Marques, M. Highly sensitive routine method for urinary 3-hydroxybenzo[a]pyrene quantitation using liquid chromatography-fluorescence detection and automated off-line solid phase extraction. Analyst 2011, 136, 1183-1191. [CrossRef]

29. Simon, P.; Lafontaine, M.; Delsaut, P.; Morele, Y.; Nicot, T. Trace determination of urinary 3-hydroxybenzo[a]pyrene by automated column-switching high-performance liquid chromatotgraphy. J. Chromatogr. B Biomed. Sci. Appl. 2000, 748, 337-348. [CrossRef]

30. Lafontaine, M.; Champmartin, C.; Simon, P.; Delsaut, P.; Funck-Brentano, C. 3-Hydroxybenzo[a]pyrene in urine of smokers and non-smokers. Toxicol. Lett. 2006, 162, 181-185. [CrossRef]

31. Richter-Brockmann, S.; Dettbarn, G.; Jessel, S.; John, A.; Seidel, A.; Achten, C. Ultra-high sensitive analysis of 3hydroxybenzo[a]pyrene in human urine using GC-APLI-MS. J. Chromatogr. B 2019, 1118-1119, 187-193. [CrossRef]

32. Sibul, F.; Burkhardt, T.; Kachhadia, A.; Pilz, F.; Scherer, G.; Scherer, M.; Pluym, N. Identification of biomarkers specific to five different nicotine product user groups: Study protocol of a controlled clinical trial. Contemp. Clin. Trials Commun. 2021, 22, 100794. [CrossRef]

33. Food and Drug Administration (FDA). Bioanalytical Method Validation-Guidance for Industry. FDA-2013-D-1020; 2018. Available online: https: / / www.fda.gov/regulatory-information/search-fda-guidance-documents/bioanalytical-method-validationguidance-industry (accessed on 6 September 2021).

34. Blaszkewicz, M.; Liesenhoff-Henze, K. Creatinine in urine [Biomonitoring Methods, 2010]. In The MAK-Collection for Occupational Health and Safety; Wiley-VCH: Weinheim, Germany, 2010; pp. 169-184.

35. Piller, M.; Gilch, G.; Scherer, G.; Scherer, M. Simple, fast and sensitive LC-MS/MS analysis for the simultaneous quantification of nicotine and 10 of its major metabolites. J. Chromatogr. B 2014, 951-952, 7-15. [CrossRef]

36. Barbeau, D.; Persoons, R.; Marques, M.; Hervé, C.; Laffitte-Rigaud, G.; Maitre, A. Relevance of urinary 3-hydroxybenzo (a) pyrene and 1-hydroxypyrene to assess exposure to carcinogenic polycyclic aromatic hydrocarbon mixtures in metallurgy workers. Ann. Occup. Hyg. 2014, 58, 579-590. [CrossRef]

37. Lafontaine, M.; Gendre, C.; Delsaut, P.; Simon, P. Urinary 3-hydroxybenzo[a]pyrene as a biomarker of exposure to polycyclic aromatic hydrocarbons: An approach for determining a biological limit value. Polycycl. Aromat. Compd. 2004, 24, 441-450. [CrossRef]

38. Li, Z.; Romanoff, L.C.; Trinidad, D.A.; Pittman, E.N.; Hilton, D.; Hubbard, K.; Carmichael, H.; Parker, J.; Calafat, A.M.; Sjödin, A. Quantification of 21 metabolites of methylnaphthalenes and polycyclic aromatic hydrocarbons in human urine. Anal. Bioanal. Chem. 2014, 406, 3119-3129. [CrossRef] [PubMed]

39. Alwis, K.U.; Blount, B.C.; Britt, A.S.; Patel, D.; Ashley, D.L. Simultaneous analysis of 28 urinary VOC metabolites using ultra high performance liquid chromatography coupled with electrospray ionization tandem mass spectrometry (UPLC-ESI/MSMS). Anal. Chim. Acta 2012, 750, 152-160. [CrossRef] [PubMed]

40. Pluym, N.; Gilch, G.; Scherer, G.; Scherer, M. Analysis of 18 urinary mercapturic acids by two high-throughput multiplex-LCMS/MS methods. Anal. Bioanal. Chem. 2015, 407, 5463-5476. [CrossRef] [PubMed]

41. Schettgen, T.; Musiol, A.; Alt, A.; Ochsmann, E.; Kraus, T. A method for the quantification of biomarkers of exposure to acrylonitrile and 1,3-butadiene in human urine by column-switching liquid chromatography-tandem mass spectrometry. Anal. Bioanal. Chem. 2009, 393, 969-981. [CrossRef] [PubMed]

42. Luo, X.; Carmella, S.G.; Chen, M.; Jensen, J.A.; Wilkens, L.R.; Le Marchand, L.; Hatsukami, D.K.; Murphy, S.E.; Hecht, S.S. Urinary Cyanoethyl Mercapturic Acid, a Biomarker of the Smoke Toxicant Acrylonitrile, Clearly Distinguishes Smokers From Nonsmokers. Nicotine Tob. Res. 2020, 22, 1744-1747. [CrossRef] [PubMed] 